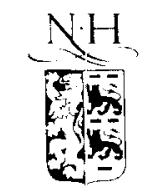

IISF:VII:R

\title{
Computation challenges in complex liquids: Entropy-driven phase transitions
}

\author{
Daan Frenkel \\ FOM Institute for Atomic and Molecular Physics, Kruislaan 407, 1098 SJ Amsterdam, The Netherlands
}

Abstract

The numerical study of complex fluids offers a challenge to numerical simulation as the systems under consideration are too large to be treated by conventional atomistic models, yet too small to be treated macroscopically. This paper sketches some of the recent trends in the numerical study of phase behavior of complex liquids. The importance of the entropy for the phase-behavior is illustrated.

Key words: Monte Carlo simulations; Colloids; Polymers; Phase transitions; Liquid crystals; Path-integrals

\section{Introduction}

Computer simulations can be used to predict the mechanical and thermal properties of existing or novel materials. Although many of the techniques to perform such calculations have been around for several decades, most simulations were limited to relatively simple atomic and molecular substances. In recent years, we have witnessed a move towards the numerical simulation of materials that are, in some sense, complex. This move was facilitated by two factors: on the one hand, the development of novel simulation techniques, such as the Car-Parrinello 'ab-initio' MD scheme [1] and, on the other hand, the ever increasing power of the available computers.

In this paper, I wish to discuss one particular class of materials with 'complex' properties, namely macro-molecular or colloidal systems. The building blocks of such materials are 'mesoscopic' rather than microscopic, and special simulation techniques are required to study their phase behaviour.

In this context, it is necessary to discuss the role of entropy in phase transitions. At first sight, there is little need for such a discussion. The second law of thermodynamics tells us that, in a closed system at equilibrium, the entropy, $S$, is at a maximum. One could therefore argue that any spontaneous phase transformation in a closed system occurs simply because it results in an increase of the entropy. However, it is more common to consider the equilibrium behavior of a system that is not isolated, but can exchange energy with its surroundings. In that case, the second law of thermodynamics implies that the system will tend to minimize its Helmholtz free energy $F=E-T S$, where $E$ is the internal energy of the system and $T$ the temperature. Clearly, a system at constant temperature can lower its 
free energy in two ways: either by increasing the entropy $S$, or by decreasing the internal energy $E$. In order to gain a better understanding of the factors that influence phase transitions, we must look at the statistical mechanical expressions for entropy. The simplest starting point is to use Boltzmann's expression for the entropy of an isolated system of $N$ particles in volume $V$ at an energy $E$,

$S=k_{B} \ln \Omega$

where $k_{B}$, the Boltzmann constant, is simply a constant of proportionality. $\Omega$ is the total number of (quantum) states that is accessible to the system. In the remainder of this paper, I shall choose my units such that $k_{B}=1$. The usual interpretation of Eq. 1 is that $\Omega$, the number of accessible states of a system, is a measure for the 'disorder' in that system. The larger the disorder, the larger the entropy. This interpretation of entropy suggests that a phase transition from a disordered to a more ordered phase can only take place if the loss in entropy is compensated by the decrease in internal energy. This statement is completely correct, provided that we use Eq. 1 to define the amount of disorder in a system. However, we also have an intuitive idea of order and disorder: crystalline solids are 'ordered', while isotropic liquids are 'disordered'. This suggests that a spontaneous phase transition from the fluid to the crystalline state can only take place if the freezing lowers the internal energy of the system sufficiently to outweigh the loss in entropy: i.e. the ordering transition is 'energy driven'. In many cases, this is precisely what happens. It would, however, be a mistake to assume that our intuitive definition of order always coincides with the one based on Eq. 1. In fact, the aim of this paper is precisely to show that many 'ordering'-transitions that are usually considered to be energydriven may, in fact, be entropy driven. At the outset, I should stress that the idea of entropydriven phase transitions is an old one. However, it has only become clear during the past few years that such phase transformations may not be interesting exceptions, but the rule!

The question that I wish to address is therefore: what classes of phase transitions can be driven by entropy alone? In order to answer this question, we must consider systems in which the internal energy is a function of temperature alone. If a first-order phase transformation takes place a constant temperature, the internal energy must remain the same. Hence the change in Helmholtz free energy is determined exclusively by the change in entropy of the system. In general, it is not obvious how to devise model systems for which the internal energy depends only on temperature. In order for this condition to hold, the partition function $Z$ of the system should factorize into a part that depends only on the temperature, $T$, and a part that depends on the density, $\rho$. For a classical $N$-body system, we can write $Z$ as

$$
Z=\frac{1}{h^{3 N} N !} \int \ldots \int d \mathbf{p}^{N} d \mathbf{q}^{N} \exp \left[-\beta H\left(\mathbf{p}^{N}, \mathbf{q}^{N}\right)\right]
$$

where $\beta=\left(k_{B} T\right)^{-1}$, while $H\left(\mathbf{p}^{N}, \mathbf{q}^{N}\right)$ denotes the Hamiltonian of the system, expressed as a function of the momenta $\mathbf{p}^{N}$ and the coordinates $\mathbf{q}^{N}$. For convenience, I have assumed that we are dealing with an atomic system. The Hamiltonian $H$ is the sum of the kinetic energy $K\left(\mathbf{p}^{N}\right)$ and the potential energy $U\left(\mathbf{q}^{N}\right)$. For classical systems, we can perform the integration over momenta in Eq. 2 analytically. It yields a factor in the partition function that depends on $T$ only. The remaining, configurational, part of the partition function is:

$Q=\frac{1}{N !} \int \ldots \int d \mathbf{q}^{N} \exp \left[-\beta U\left(\mathbf{q}^{N}\right)\right]$.

In general, $Q$ will be a function of $N, V$ and $T$. We are interested in the case that $Q$ does not depend on $T$. It would seem that this is not possible, because $Q$ depends on $\beta$. However, if we limit our attention to hard-core potentials, i.e. potential energy functions that are (depending on the value of $\mathbf{q}^{N}$ ) either 0 or $\infty$, then $Q$ is indeed independent of $\beta$. It is easy to see that, in that case, the average potential energy of the system

$$
\langle U\rangle \equiv-\frac{\partial \ln Q}{\partial \beta}=0 .
$$


The average energy of a hard-core system is therefore simply equal to the average kinetic energy $\langle K\rangle$, which is a function of the temperature only. As the internal energy of a hard-core system is constant at constant temperature, any phase transformation in such a system takes place only because this results in an increase in entropy.

Now that we have defined a class of model systems for which entropy is the only driving force behind spontaneous phase transformations, we wish to find out what kinds of phase such a model system can exhibit.

\section{2. (Liquid) crystals}

In this section, I shall briefly review what we now know about the effect of entropy on the formation of partially ordered liquids ('liquid crystals') and crystalline solids. The earliest example of an entropy-driven ordering transition is described in a classic paper of Onsager [2], on the isotropic-nematic transition in a (three-dimensional) system of thin hard rods. Onsager showed that, on compression, a fluid of thin hard rods of length $L$ and diameter $D$ must undergo a transition from the isotropic fluid phase, where the molecules are translationally and orientationally disordered, to the nematic phase. In the latter phase, the molecules are translationally disordered, but their orientations are, on average, aligned. This transitions takes place at a density such that $(N / V) L^{2} D=\mathscr{O}(1)$. Onsager considered the limit $L / D \rightarrow \infty$. In this case, the phase transition of the hard-rod model can be found exactly (see e.g. [3]). At first sight it may seem strange that the hard rod system can increase its entropy by going from a disordered fluid phase to an orientationally ordered phase. Indeed, due to the orientational ordering of the system, the orientational entropy of the system decreases. However, this loss in entropy is more than offset by the increase in translational entropy of the system: the available space for any one rod increases as the rods become more aligned. In fact, we shall see this mechanism returning time-and-again in ordering transitions of hard-core systems: the entropy decreases because the density is no longer uniform in orientation or position, but the entropy increases because the free-volume per particle is larger in the ordered than in the disordered phase.

The most famous, and for a long time controversial, example of an entropy-driven ordering transition is the freezing transition in a system of hard spheres. This transition had been predicted by Kirkwood in the early fifties [4] on basis of an approximate theoretical description of the hardsphere model. As this prediction was quite counter-intuitive and not based on any rigorous theoretical results, it met with wide-spread skepticism until Alder and Wainwright [5] and Wood and Jacobson [6] performed numerical simulations of the hard-sphere system that showed direct evidence for this freezing transition. Even then, the acceptance of the idea that freezing could be an entropy driven transition, came only slowly [7]. However, by now, the idea that hard spheres undergo a first-order freezing transition is generally accepted. And, although the hardsphere model was originally devised as an idealized and highly unrealistic model of an atomic fluid, it is now realized that this model provides a good description of certain classes of colloidal systems.

The next step in this sequence came in the mid-eighties when computer simulations [8] showed that hard-core interactions alone could also explain the formation of more complex liquid crystals. In particular, it was found that a system of hard sphero-cylinders (i.e. cylinders with hemi-spherical caps) can form a smectic liquid crystal, in addition to the isotropic liquid, the nematic phase the crystalline solid. In the smectic (A) phase, the molecules are orientationally ordered but, in addition, the translational symmetry is broken: the system exhibits a one-dimensional density-modulation. Subsequently, it was found that some hard-core models could also exhibit columnar ordering [9]. In the latter case, the molecules assemble in liquid-like stacks, but these stacks order to form a two-dimensional crystal. In summary, hard-core interaction can induce orientational ordering and one-, two- and three-dimensional positional ordering. This is rather surprising because, in particular for the smectic and 
the columnar phase, it was generally believed that their formation required specific energetic interactions.

\section{Depletion flocculation}

Let us next consider a slightly more complex example of an entropy-driven phase separation in a binary mixture, namely polymer-induced flocculation of colloids. Experimentally, it is well known that the addition of a small amount of free, non-adsorbing polymer to a colloidal suspension induces an effective attraction between the colloidal particles and may even lead to coagulation. This effect has been studied extensively [11] and is well understood, at least qualitatively. As in the example discussed above, the polymer-induced attraction between colloids is an entropic effect: when the colloidal particles are close together, the total number of accessible polymer conformations is larger than when the colloidal particles are far apart. However, although the physical mechanism responsible for polymer-induced coagulation is understood qualitatively, a quantitative description of this phenomenon is difficult. This is so because the polymer-induced attraction between the colloidal particles is non-pairwise additive. Moreover, it depends both on the osmotic pressure of the polymer and on the concentration of the colloid. Yet, in the theoretical description of polymer-induced clustering [12], the effect of the polymer is usually replaced by an effective, density-independent, pair-wise additive attraction between the colloidal particles. However, in this case, no analytical evaluation of the (grand-canonical) partition function is possible, even when one considers only the very simplest model, viz. that of a mixture of hard-core colloidal particles with ideal chain molecules with conformations that are restricted to a lattice. In this case, it would clearly be desirable to carry out 'exact' numerical simulations to investigate the phase behavior. Yet, the computational problems are still formidable. What is required is a numerical scheme that samples the positions of the colloidal particles while averaging over all possible conformations of a large (and fluctuating) number of chain molecules. The 'conventional' Monte Carlo schemes to simulate lattice models of polymer systems [10] would be woefully inadequate for such a calculation.

Fortunately, it is possible to construct a rigorous and efficient Monte Carlo scheme to study this model. Our approach relies on the fact that we can recursively compute the partition function of an ideal (non-self avoiding) chain on a lattice in an arbitrary external potential $[13,14]$. This is most easily seen by considering a chain of length $l-1$ on a lattice. For convenience, we assume that the external potential is either zero or infinite. The total number of accessible ideal chain conformations that terminate on lattice site $i$ is denoted by $\omega_{l-1}(i)$. The total partition function $\Omega_{l-1}$ is equal to $\sum_{i} \omega_{l-1}(i)$, where the sum runs over all lattice sites. The total number of chains of length $l$ that terminate on site $i$ is clearly equal to the sum of the total number of chains of length $l-1$ that terminate on any of the neighbors of $i$, multiplied by the Boltzmann factor associated with site $i$. Using such a recursive scheme, we can compute exactly $\Omega_{l}$, the partition function of a single ideal polymer or arbitrary length $l$ on a lattice, in an arbitrary external potential. This scheme can be used as a starting point to study self-avoiding polymers $[15,16]$, but for the present purpose we limit ourselves to ideal polymers. Up to this point we have not specified the nature of the 'external' potential. We now assume that this potential is due to the presence of $N$ hard, spherical colloidal particles each of which occupies many lattice sites. The polymer partition function clearly depends on the coordinates $\mathbf{r}^{N}$ of the colloidal particles: $\Omega_{l}\left(\mathbf{r}^{N}\right)$. The configurational part of the partition function of the system of $N$ colloids plus one polymer of length $l$ in volume $V$ is then given by:

$Z(V, N, 1)=\int_{V} d \mathbf{r}^{N} e^{-U_{h s}\left(\mathbf{r}^{N}\right)}\left(\Omega_{l}\left(\mathbf{r}^{N}\right)\right)$

where $U_{h s}\left(\mathbf{r}^{N}\right)$ denotes the hard-sphere interaction. Next, we make use of the fact that we are considering ideal polymers. In that case we can immediately write down the corresponding parti- 
tion function for $N$ colloids and $M$ ideal polymers:

$Z(V, N, M)=\int_{V} d \mathbf{r}^{N} e^{-U_{h s}\left(\mathbf{r}^{N}\right)}\left(\Omega_{l}\left(\mathbf{r}^{N}\right)\right)^{M} / M !$,

where the factor $1 / M !$ accounts for the fact that the polymers are indistinguishable. Using Eq. 6 it is straightforward to transform to an ensemble where the polymer chemical potential (i.e. the osmotic pressure) is kept fixed. The corresponding grand-canonical partition function is given by:

$$
\begin{aligned}
& \exists(V, N, \mu) \\
& \quad=\sum_{M=0}^{\infty} e^{M \mu_{c h}} Z(V, N, M) / M ! \\
& \quad=\int_{V} d \mathbf{r}^{N} e^{-U_{h s}\left(\mathbf{r}^{N}\right)} \sum_{M=0}^{\infty} e^{M \mu_{c h}}\left(\Omega_{l}\left(\mathbf{r}^{N}\right)\right)^{M} / M ! \\
& \quad=\int_{V} d \mathbf{r}^{N} e^{-U_{h s}\left(\mathbf{r}^{N}\right)} e^{z \Omega_{l}\left(\mathbf{r}^{N}\right)} .
\end{aligned}
$$

In the last line of Eq. 7, we have introduced the polymer fugacity $z \equiv e_{c h}^{\mu}$, where $\mu_{c h}$ denotes the chemical potential of the chain molecules.

The important point to note is that Eq. 7 allows us to evaluate the properties of the colloidal particles in osmotic equilibrium with a polymer reservoir. In particular, it shows that we can perform Monte Carlo sampling of the colloidal particles. The polymers only affect $U_{e f f}\left(\mathbf{r}^{N}\right)$, the effective interaction between the colloidal particles:

$U_{e f f}\left(\mathbf{r}^{N}\right) \equiv U_{h s}\left(\mathbf{r}^{N}\right)-z \Omega_{l}\left(\mathbf{r}^{N}\right)$.

$z \Omega_{l}\left(\mathbf{r}^{N}\right)$ measures the entropic interaction between the colloids due to all possible polymer conformations. This entropic interaction is, in principle, not pairwise additive. In fact, it is shown in [14], that for all but the shortest chain molecules, this non-additivity of the polymer-induced interaction between the colloids, has a pronounced effect on the structure and stability of the mixture. For more details, we refer the reader to [14].

\section{4. 'Entropy' in quantum phase-transitions}

Finally, I wish to discuss a more speculative, and somewhat exotic, example of an entropydriven phase transition, namely the demixing of a system of ideal point fermions ('non-interacting electrons') and classical fluid particles. The analogy between this system and the previous ones rests on the fact that, in Feynman's path-integral formulation of quantum mechanics, there is an isomorphism between the partition-function of a $d$-dimensional quantum-mechanical system, and that of $d+1$-dimensional, 'polymer' system. Ideal quantum particles in $d$ dimensions can be described by ideal, directed polymers in $d+1$ dimensions. This suggests that we may expect that mixtures of ideal quantum particles and atoms (effectively, hard spheres) behave much like a mixture of ideal polymers and colloids. In fact, the situation in the quantum case is complicated by the fact that one must take exchange into account. This is relatively straightforward in the case of numerical simulations of bosons [18], but it creates serious problems for fermions (the 'sign'-problem). However, in [19], it is shown how, at least for a mixture of atoms and ideal fermions, these problems may be overcome. Alavi and Frenkel [19] show that it is, in fact, possible to evaluate numerically (but exactly) the grandcanonical partition function of ideal fermions in the presence of arbitrary 'obstacles' (in particular, atoms). This makes it possible to perform Monte Carlo sampling of the atomic positions in the presence of the fermion 'bath'. Here, I will not discuss the technical aspects of these simulations. Rather, I will summarize the main conclusion, which is that there is indeed strong evidence for a demixing transition as the chemical potential of the fermions is increased beyond a certain critical value. Again, there are no attractive forces in this system. Hence, this fermion-induced phase separation appears to be yet another example of 'attraction through repulsion'. For more details, the reader is referred to [19]. It is amusing to note that the phase-separation in a mixture of ideal fermions and hard-spherical 'atoms' may have an intriguing counterpart in planetary astronomy, namely in the structure of the interior 
of Saturn. It is thought that the core of Saturn, which is a hydrogen-helium planet, consists of nearly pure helium [20], whilst at the conditions relevant to the interior of Saturn $\left(T \approx 10^{4} \mathrm{~K}\right.$, $P \approx 1-10$ Mbar) the hydrogen probably is metallic. If we view the helium as consisting of 'hardspheres', and the metallic hydrogen as a nearly free electron gas (in which the hydrogen nuclei play the role of a neutralising background), we might expect on the basis of the calculations in [19] that such a mixture would phase-separate.

\section{Acknowledgments}

This work is part of the research program of FOM (Foundation for Fundamental Research on Matter), and is supported by The Netherlands Organisation for Scientific Research (NWO). I gratefully acknowledge the contributions of Ali Alavi, Ard Louis and Evert Jan Meijer.

\section{References}

[1] See the contribution of M.J. Gillan in this issue.

[2] L. Onsager, Proc. NY. Acad. Sci. 51 (1949) 627.

[3] R.F. Kayser and H.J. Raveche, Phys. Rev. Al7 (1978) 2067.

[4] J.E. Kirkwood in Phase Transformations in Solids, R. Smoluchowski, J.E. Mayer and W.A. Weyl, eds. (Wiley, New York, 1951) 67.

[5] B.J. Alder and T.E. Wainwright, J. Chem. Phys. 27 (1957) 1208.
[6] W.W. Wood and J.D. Jacobson, J. Chem. Phys. 27 (1957) 1207.

[7] The Many-Body Problem, J.K. Percus, ed. (Interscience, New York, 1963).

[8] D. Frenkel, H.N.W. Lekkerkerker and A. Stroobants, Nature 332 (1988) 822.

[9] J.A.C. Veerman and D. Frenkel, Phys. Rev. A45 (1992) 5633.

[10] K. Kremer and K. Binder, Comput. Phys. Rep. 7 (1988) 259.

[11] For a review and other references see D.H. Napper, Polymeric Stabilization of Colloidal Dispersions (Academic Press, New York, 1983), Ch. 16.

[12] See, e.g. B. Vincent, J. Edwards, S. Emmett and R. Groot, Colloids Surfaces 31 (1988) 267.

[13] D. Frenkel, J. Phys. Condens. Matter 2 (1990) SA265.

[14] E.J. Meijer and D. Frenkel, Computer simulation of polymer-induced clustering of colloids, Phys. Rev. Lett. 67 (1991) 1110.

[15] G.C.A.M. Mooij and D. Frenkel, Mol. Phys. 74 (1991) 41.

[16] D. Frenkel, Physica A176 (1991) 54.

[17] R.P. Feynman and A.R. Hibbs, Quantum Mechanics and Path Integrals, McGraw-Hill (1965). The analogy between path-integrals and polymers is explained in: D. Chandler, P.G. Wolynes, J. Chem. Phys. 74 (1981) 7.

[18] D.M. Ceperley and E.L. Pollock, Phys. Rev. Lett. 56 (1986) 351.

[19] A. Alavi and D. Frenkel, J. Chem. Phys. 97 (1992) 9249.

[20] D.J. Stevenson and E.E. Salpeter, Astrophys. J. Suppl. Series 35 (1977) 221.

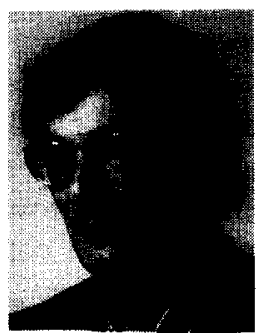

Daan Frenkel (1948) obtained a Ph. D. in experimental Physical Chemistry at the University of Amsterdam. He spent two years as a postdoctoral fellow at UCLA. Subsequently, he worked at Shell Research and the Physics Department of University of Utrecht. Since 1987, he is groupleader Computational Physics at the FOM Institute in Amsterdam and professor of Computational Physical Chemistry at Utrecht University. 